\title{
The cortical representation of the speech envelope is earlier for audiovisual speech than audio speech
}

\author{
Michael J. Crosse ${ }^{1,2}$ and Edmund C. Lalor ${ }^{1,2,3}$ \\ ${ }^{1}$ School of Engineering, Trinity College Dublin, Dublin, Ireland; ${ }^{2}$ Trinity Centre for Bioengineering, Trinity College Dublin, \\ Dublin, Ireland; and ${ }^{3}$ Trinity College Institute of Neuroscience, Trinity College Dublin, Dublin, Ireland
}

Submitted 25 September 2013; accepted in final form 29 December 2013

Crosse MJ, Lalor EC. The cortical representation of the speech envelope is earlier for audiovisual speech than audio speech. $J$ Neurophysiol 111: 1400-1408, 2014. First published January 8, 2014; doi:10.1152/jn.00690.2013.- - Visual speech can greatly enhance a listener's comprehension of auditory speech when they are presented simultaneously. Efforts to determine the neural underpinnings of this phenomenon have been hampered by the limited temporal resolution of hemodynamic imaging and the fact that EEG and magnetoencephalographic data are usually analyzed in response to simple, discrete stimuli. Recent research has shown that neuronal activity in human auditory cortex tracks the envelope of natural speech. Here, we exploit this finding by estimating a linear forward-mapping between the speech envelope and EEG data and show that the latency at which the envelope of natural speech is represented in cortex is shortened by $>10 \mathrm{~ms}$ when continuous audiovisual speech is presented compared with audio-only speech. In addition, we use a reverse-mapping approach to reconstruct an estimate of the speech stimulus from the EEG data and, by comparing the bimodal estimate with the sum of the unimodal estimates, find no evidence of any nonlinear additive effects in the audiovisual speech condition. These findings point to an underlying mechanism that could account for enhanced comprehension during audiovisual speech. Specifically, we hypothesize that low-level acoustic features that are temporally coherent with the preceding visual stream may be synthesized into a speech object at an earlier latency, which may provide an extended period of low-level processing before extraction of semantic information.

multisensory integration; analysis-by-synthesis; latency; EEG; TRF

DURING NATURAL, EVERYDAY CONVERSATION, we routinely process speech using both our auditory and visual systems. Indeed, visual information can improve speech comprehension in both noisy (Sumby and Pollack 1954) and noise-free environments (Reisberg et al. 1987). This perceptual improvement may be driven largely by our ability to utilize the temporal precedence of visual speech to predict incoming auditory information (Arnal et al. 2009; Besle et al. 2004; van Wassenhove et al. 2005). Although we easily integrate continuous audio and visual speech, the temporal delay between the unimodal streams varies with speaker and content by up to several hundred milliseconds (Chandrasekaran et al. 2009), making it an intrinsically complex process. Examining the temporal dynamics of audiovisual (AV) speech processing is, therefore, essential to understanding how information from the two modalities is integrated into one perceptual representation.

The limited temporal resolution of functional MRI (fMRI) makes it unsuitable for investigating the rapid dynamics of

Address for reprint requests and other correspondence: E. C. Lalor, Printing House, Trinity College Dublin, College Green, Dublin 2, Ireland (e-mail: edlalor@tcd.ie). ongoing speech. In contrast, EEG and magnetoencephalography (MEG) offer excellent temporal resolution, rendering them more suitable methodologies for addressing this subject. However, the standard technique for investigating sensory processing is to present discrete stimuli repeatedly and to derive an average response. In the context of speech, this has largely constrained researchers to examining the processing of individual syllables. This approach overlooks the complex dynamics of speech, which is continuous in nature, and severely constrains the stimuli in terms of their semantic content. However, within this context, many studies have shown important effects of visual input, including the attenuation of key auditory processing indices (Arnal et al. 2009; Besle et al. 2004; Klucharev et al. 2003; Pilling 2009; Stekelenburg and Vroomen 2007; van Wassenhove et al. 2005).

Several of these studies have also reported shorter auditory response latencies when auditory and visual syllables are presented concurrently (Arnal et al. 2009; Stekelenburg and Vroomen 2007; van Wassenhove et al. 2005). This effect has been shown not just for congruent AV syllables, but also for discrete nonspeech stimuli (Stekelenburg and Vroomen 2007), discrete incongruent speech stimuli (Arnal et al. 2009; Stekelenburg and Vroomen 2007), and discrete monkey vocalizations (Chandrasekaran et al. 2013). However, to date, no evidence has been advanced for such a shift in the case of natural continuous speech in humans.

Although the latency effects reported to discrete stimuli fit with the fact that visual speech precedes auditory speech (Chandrasekaran et al. 2009), these effects can complicate the issue of examining nonlinear additive effects between the responses evoked by different modes of speech, such as the attenuation effects mentioned above. This is because the dominant components of event-related potentials (ERPs) are generated by multiple temporally overlapping sources and, hence, are likely composed of several subcomponents (Di Russo et al. 2005). A shift in the latency of any one of these contributions can result in both latency and amplitude changes in the measured ERP component. Indeed, latency shifts in the ERP components can also have an impact on temporally adjacent ERP components, affecting their amplitude (Luck 2004). The reverse is also true in that amplitude differences between components or subcomponents could masquerade as latency shifts when examining ERPs, an issue that has been raised before (Stekelenburg and Vroomen 2007). New approaches are necessary to disentangle latency effects from amplitude effects in human $\mathrm{AV}$ speech processing.

This study seeks to investigate this issue using two complementary methods that exploit the fact that changes in cortical 
activity track the dynamic changes in the speech stimulus (Lalor and Foxe 2010; Millman et al. 2013; Nourski et al. 2009). Specifically, we examine the effects of visual speech on linear response function measures that index how the amplitude envelope of natural speech maps forward to the recorded EEG (Lalor and Foxe 2010). We also exploit a reversemapping approach between the EEG and the amplitude envelope (Mesgarani et al. 2009) to quantify multisensory amplitude effects in a manner that is relatively free from the confounds of latency effects. In addition to allowing us to examine continuous AV speech processing, our assumption of a linear relationship between the envelope and EEG data has two methodological benefits. First, it minimizes the contribution of the visual stimulus during the time frame of the auditory response because, unlike discrete stimuli, the dynamics of the visual stimulus are not tightly time-locked to those of the audio stimulus. As such, we can compare neuronal responses to audio-only (A) speech with those from AV speech in terms of how just the auditory stream was represented. Second, unlike the ERP, it produces response measures that are indicative of feedforward activity in early auditory cortex (Lalor et al. 2009). We anticipate that these two properties of the approach will allow us to search for latency effects in auditory cortex in the context of continuous stimuli and to provide greater clarity on the issue of latency shifts vs. amplitude effects than can be gleaned from examining ERPs derived from discrete stimuli.

\section{METHODS}

Subjects. Fourteen right-handed volunteers aged between 20 and 35 yr participated with informed, written consent ( 4 women; mean age, $25.4 \mathrm{yr}$ ). All subjects were free of neurological diseases and had self-reported normal hearing and normal or corrected-to-normal vision. The experiment was undertaken in accordance with the Declaration of Helsinki and was approved by the Faculty Ethics Committee of the Faculty of Health Sciences at Trinity College Dublin, Ireland.

Stimuli and experimental procedure. The stimuli were drawn from a collection of videos featuring a native speaker of English (male). The speaker's face was positioned at the center of the camera shot, which alternated between medium close-up and close-up while the speaker addressed the camera at all times. There was no background movement or noise. Using VideoPad Video Editor (NCH Software), 7 videos were truncated to $120 \mathrm{~s}$ in length and rendered into $640-\times$ 360-pixel movies with a digitization rate of 29.97 frames per second ( 1 frame $=33.37 \mathrm{~ms}$ ). The stereo soundtracks were digitized at 44.1 $\mathrm{kHz}$ with 16-bit resolution. Stimulus presentation was conducted using software by Presentation (Neurobehavioral Systems) and delivered using a 19-in. cathode-ray tube monitor and Sennheiser HD 650 headphones. Each of the 7 videos was presented in A, visual-only (V), and $\mathrm{AV}$ format, the order of which was randomized between blocks. Subjects were instructed to attend to the auditory stimulus while maintaining visual fixation on the speaker's mouth for the V and AV conditions and on a gray crosshair for the A condition. Subjects were positioned $70 \mathrm{~cm}$ from the screen of the monitor in a dark room and instructed to keep eye blinks and all other motor activity to a minimum.

EEG data acquisition and preprocessing. EEG data were recorded at 130 locations (128 scalp and left and right mastoids) along with triggers indicating the start and end of each audio passage using an ActiveTwo system (BioSemi, Amsterdam, The Netherlands). The data were low-pass filtered online below $134 \mathrm{~Hz}$ and digitized at a rate of $512 \mathrm{~Hz}$. Subsequent processing was conducted using MATLAB (MathWorks, Natick, MA); the data were digitally band-pass filtered between 0.5 and $25 \mathrm{~Hz}$ and referenced to the average of the mastoid channels. Bad channels were detected and recalculated by splineinterpolating the surrounding good channels. These channels were identified as those with a SD that was $>3$ times (or $<1 / 3$ of) the SD of the surrounding channels.

EEG data analysis. Two complementary methods were employed for quantifying the representation of the speech envelope in the EEG data. The 1st method describes the linear forward-mapping from the stimulus envelope to each of the 128 scalp electrodes, known as the temporal response function (TRF; Lalor and Foxe 2010; Lalor et al. 2009). The 2 nd method describes the linear multivariate mapping from all 128 electrodes simultaneously back to the stimulus envelope, allowing reconstruction of the stimulus from unseen data (Mesgarani et al. 2009). The next 2 sections describe each of these methods in further detail. Please refer to Fig. 1 for an overview of this process.

TRF estimation. Based on the assumption that the EEG response (R) to the speech on each separate channel $(n)$ consisted of a convolution of the broadband speech envelope (S) with an unknown, channel-specific TRF $(w)$, the response model can be represented in discrete time as:

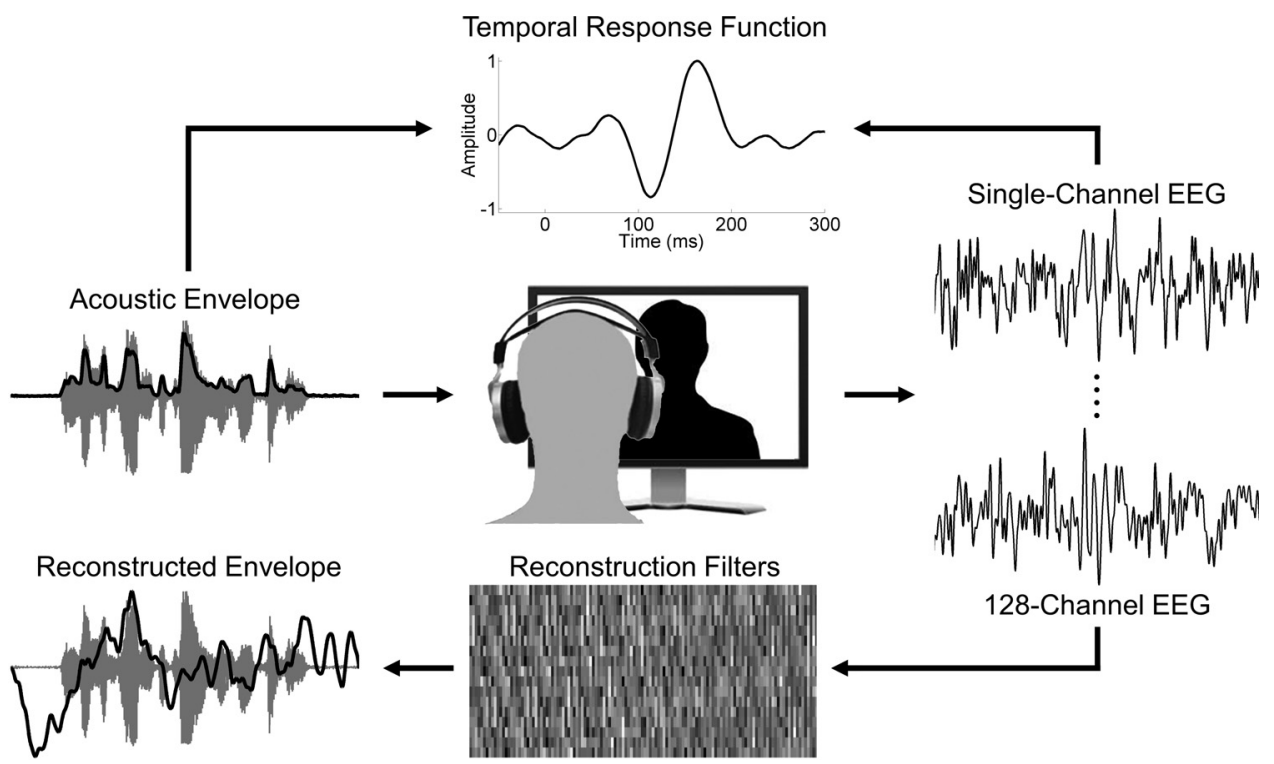

Fig. 1. Linear forward- and backward-mapping techniques. The temporal response function (TRF), which represents the mapping from the acoustic envelope to the EEG data at each channel, was calculated using a linear least-squares approach. The latencies of defined TRF components were measured and compared between audio-only (A) and audiovisual (AV) speech. All 128 channels of EEG were used simultaneously to reconstruct an estimate of the acoustic envelope from unseen EEG data using a reverse-mapping technique. Reconstruction accuracy was measured by calculating the Pearson correlation between the reconstructed estimate and the original envelope. 


$$
\mathrm{R}(t, n)=\sum_{\tau} w(\tau, n) \mathrm{S}(t-\tau)+\varepsilon(t, n), \quad \text { for } n=1,2, \ldots, 128,
$$

where $\varepsilon$ is the residual response not explained by the model. A TRF can be compared with a filter that describes the linear transformation of the continuous speech stimulus to the continuous EEG signal by the brain. Each channel-specific TRF $w(\tau, n)$ was calculated at all lags $(\tau)$ between -100 and $400 \mathrm{~ms}$ relative to the stimulus onset, similar to the time interval typically used to view an auditory evoked potential (AEP). First, the envelope $S$ was calculated using a Hilbert transform and then downsampled from $44.1 \mathrm{kHz}$ to $512 \mathrm{~Hz}$ after applying a zero-phase-shift antialiasing filter. Then, an autocovariance matrix for the speech envelope was calculated by computing the sliding dot product of $S_{t}$ and $S_{t}^{T}$ across all time points and then dividing by the number of time points. A sliding dot product of $\mathrm{S}_{t}$ and $\mathrm{R}_{t}(n)$ was then computed, and this was multiplied by the inverse of the stimulus autocovariance matrix. A regularization term was included to reduce the variance of the estimate by quadratically penalizing the difference between each two neighboring terms of $w$. This procedure can be written:

$$
\begin{gathered}
w=\left\langle\mathrm{S}_{t} \mathrm{~S}_{t}^{T}+\lambda M\right\rangle^{-1}\left\langle\mathrm{~S}_{t} \mathrm{R}_{t}\right\rangle, \\
\text { where } M=\left[\begin{array}{cccccc}
1 & -1 & & & & \\
-1 & 2 & -1 & & & \\
& -1 & 2 & -1 & & \\
& & \ddots & \ddots & \ddots & \\
& & & -1 & 2 & -1 \\
& & & & -1 & 1
\end{array}\right]
\end{gathered}
$$

and where $\langle\cdot\rangle$ indicates an average over time. The value of $\lambda(4.4 \times$ $10^{-3}$ ) was empirically determined to preserve component amplitude. See Lalor and Foxe (2010) and Lalor et al. (2009) for further details.

As alluded to above, the linear assumption underlying the estimation of the TRF has implications for its interpretation. This assumption of a linear relationship between speech amplitude and EEG likely results in a response measure reflective of feedforward activity in a subset of auditory cortical cells (Lalor et al. 2009). This is in contrast to the challenge involved in disambiguating the myriad feedforward, lateral, and feedback contributions to the time-locked average AEP (Di Russo et al. 2005). As such, we expect it to provide a clearer insight into the timing of activation of auditory cortex under $\mathrm{A}$ and AV conditions than might be possible with the AEP.

Stimulus reconstruction analysis. The relationship between the input speech dynamics and the multichannel EEG can also be captured using a multivariate analysis that offers certain advantages. Specifically, by weighting the relative contribution from each electrode simultaneously using a multivariate linear filter that incorporates the channel covariance structure in the estimation of the impulse response, one can attempt to reconstruct the speech stimulus from the EEG data using all of the available information across the scalp at each point in time. This method can result in a significant quantitative improvement in the input-output mapping relative to the TRF approach mentioned above.

To do this, a linear multivariate filter $(g)$ was derived that describes an optimal mapping from the multichannel EEG data R back to the envelope S. Similar to the TRF estimation above, the filter $g(\tau, n)$ can be calculated using normalized reverse correlation:

$$
g=\left\langle\mathrm{R}_{t} \mathrm{R}_{t}^{T}\right\rangle^{-1}\left\langle\mathrm{R}_{t} \mathrm{~S}_{t}^{T}\right\rangle .
$$

Given new EEG data, one can then use this filter to reconstruct an estimate of the speech stimulus that was presented during the recording of that data. In the case of $N$ channel EEG data, this can be written as:

$$
\hat{S}(t)=\sum_{n=1}^{N} \sum_{\tau} g(\tau, n) \mathrm{R}(t-\tau, n),
$$

where $\hat{S}$ denotes the estimated stimulus. See Mesgarani et al. (2009) for further details.

In this study, EEG at lags from 0 to $250 \mathrm{~ms}$ was used for stimulus reconstruction. The EEG and envelope were downsampled to $64 \mathrm{~Hz}$ to reduce processing time. For each condition, six of the seven filters were averaged and used to reconstruct the stimulus from the remaining seventh run in a leave-one-out cross-validation. The reconstruction accuracy was measured by calculating the correlation coefficient (Pearson $r$ ) between the reconstructed and original stimulus envelope. Analogous to the methods used in previous ERP research (Molholm et al. 2002), we aimed to quantify nonlinear additive multisensory contributions by comparing the AV reconstructions with the sum of the unimodal (A and $\mathrm{V}$ ) reconstructions.

\section{RESULTS}

Latency of TRF components. Unsurprisingly, given the assumption underlying our TRF analysis, we observed robust TRFs for both the A and AV conditions that displayed topographic scalp distributions that were typical of auditory cortical activity. These TRFs displayed two dominant components in the A and AV condition: a negativity at $\sim 100 \mathrm{~ms}$ and a positivity at $\sim 170 \mathrm{~ms}$. On all electrodes where these TRFs were apparent, we observed a marked shortening in the latency of the negative component for the AV condition compared with the A condition (Fig. 2A) even at the single-subject level (Fig. 2B). Across all subjects, this facilitation was of the order of $10 \mathrm{~ms}$. For example, at frontocentral electrode Fz, the mean latency of the early negative component was reduced by 11.4 $\mathrm{ms}$ in the AV condition to $98.8 \mathrm{~ms}$ (SD $8.9 \mathrm{~ms}$ ) compared with $110.2 \mathrm{~ms}$ (SD $13.4 \mathrm{~ms}$ ) in the A condition, which was significant across all 14 subjects $\left(P=2.7 \times 10^{-3}\right.$, paired $t$-test $)$. There was no significant shift in the later positive component ( $P=0.44$, paired $t$-test), which had mean latencies of $169.5 \mathrm{~ms}$ (SD $14.7 \mathrm{~ms}$ ) for the A condition and $165.5 \mathrm{~ms}$ (SD $16.9 \mathrm{~ms}$ ) for the AV condition (Fig. $2 A$ ) despite the fact that such a shift was visible in those single subjects whose TRFs displayed the highest signal-to-noise ratios (Fig. 2B).

Of course, an apparent latency shift between AV and A could manifest due to additional contribution to the TRF from the visual stimulus in the AV condition. This seemed unlikely given the lack of any large components in the TRF corresponding to the $\mathrm{V}$ condition relative to both the A and AV TRFs during the time frame of the negative component (Fig. 2A). Indeed, the V TRF amplitudes in this time interval were not noticeably larger than the prestimulus baseline. We confirmed this at channel Fz by comparing the root-mean-square amplitude of the $\mathrm{V}$ condition TRF over the prestimulus interval $(-100$ to $0 \mathrm{~ms})$ with that over the interval 0 to $300 \mathrm{~ms}$ and finding no significant difference ( $P=0.61$, paired $t$-test). Despite this, we wished to investigate the issue further and did by summing the TRF measures from the A and $\mathrm{V}$ conditions and comparing that with the TRF from the AV condition (Fig. 3). In the $\mathrm{A}+\mathrm{V} \mathrm{TRF}$, the mean latency of the negative component occurred at $113.6 \mathrm{~ms}$ (SD 10.6), which was not significantly different from that of the A condition $(P=0.37$, paired $t$-test) but was significantly later than that of the AV condition $\left(P=2.2 \times 10^{-5}\right.$, paired $t$-test $)$. Importantly, this demonstrates that the facilitation we observe in the AV con- 
A

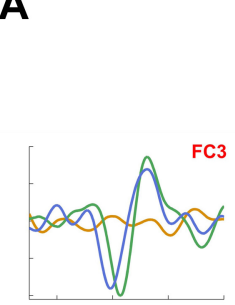

\section{Grand Average}
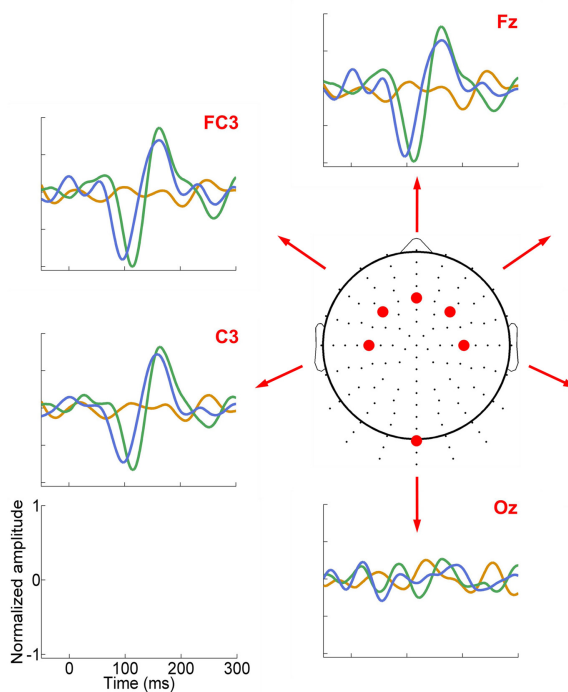

B
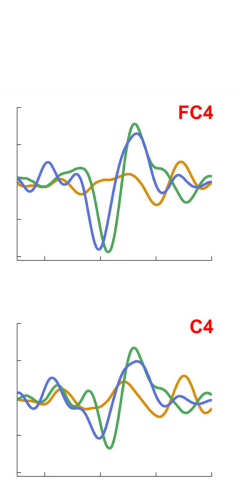

1

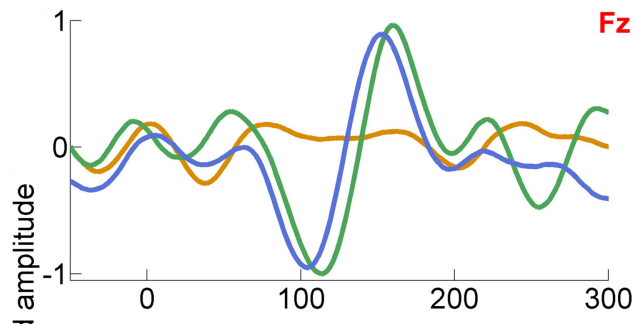

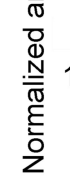

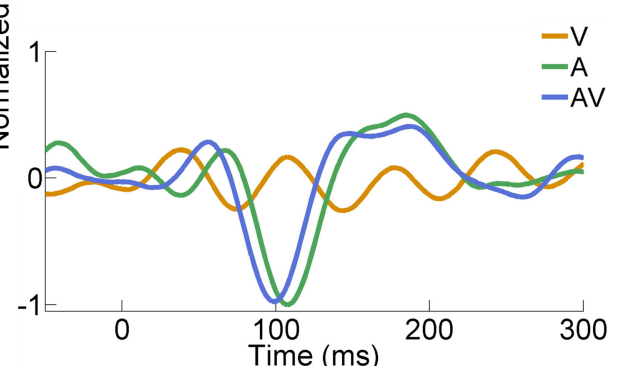

Fig. 2. Comparison of bimodal and unimodal TRFs. A: grand-average TRFs calculated at several frontocentral sites (C3, FC3, Fz, FC4, and C4) and at occipital site (Oz) for visual-only (V), A, and AV speech. At each of the frontocentral sites, the negative component of the AV response (blue trace) is noticeably earlier than that of the A response (green trace), whereas the TRFs calculated from the V data (orange trace) are considerably reduced in amplitude. No significant TRF measure was calculated at occipital location $\mathrm{Oz}$ for any of the 3 conditions. B: TRF measures calculated at Fz for 2 subjects who displayed the highest signal-to-noise ratios. The latency shift is still clearly visible at the single subject level and is even evident in the later positive component.

dition cannot simply be explained by the linear superposition of the $\mathrm{A}$ and $\mathrm{V}$ responses.

To support further our conclusion that what we observed was a genuine shift in the latency of the negative component, rather than an artifact caused by the addition of visual activity to the TRF in the AV condition, we examined the topographic distribution of the negative and positive components between

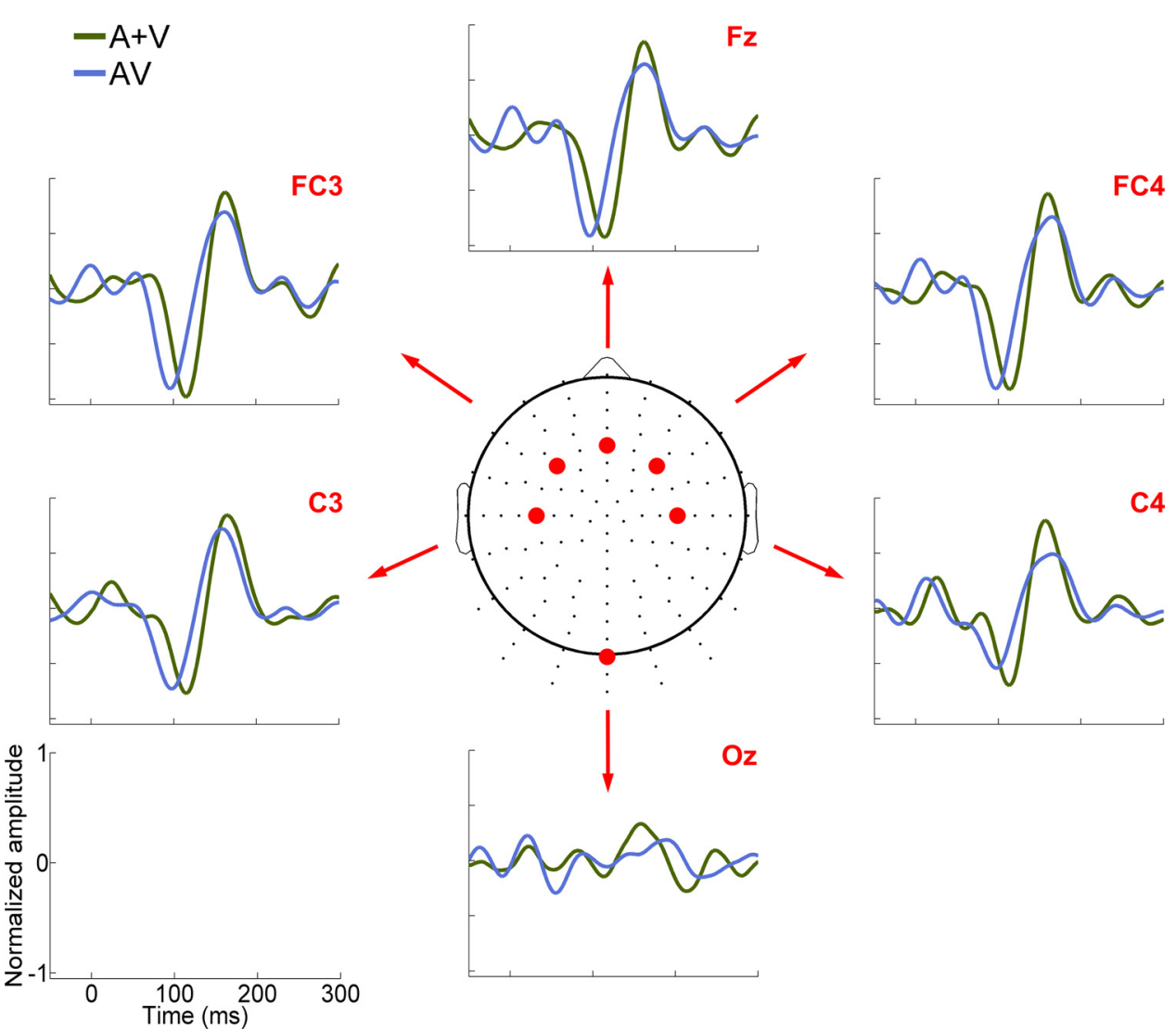

the A and AV conditions (Fig. 4; Delorme and Makeig 2004). Using a nonparametric randomization test (Murray et al. 2008), we found no statistical difference between these distributions for either the negative component $[P=0.63$, time-course ANOVA (TANOVA)] or the positive component $(P=0.68$, TANOVA), suggesting that they are likely to be predominantly generated by the same cortical sources. This is further illus-
Fig. 3. Comparison of bimodal and summed unimodal TRFs. Grand-average TRFs were calculated at the same frontocentral sites (C3, FC3, Fz, FC4, and C4) and occipital site $(\mathrm{Oz})$ for $\mathrm{AV}$ speech and for a summation of the $A$ and $V$ responses $(A+V)$. The negative component of the AV response (blue trace) remains earlier than that of the $\mathrm{A}+\mathrm{V}$ response (brown trace). Again, no significant TRF measure was calculated at occipital location $\mathrm{Oz}$ for either of the 2 conditions. 
Fig. 4. Topographical distributions of dominant TRF components for A and AV speech. The distribution of the negative component for $\mathrm{A}(\sim 110 \mathrm{~ms})$ and $\mathrm{AV}(\sim 100 \mathrm{~ms})$ speech is shown in the top left and top middle, respectively, whereas the distribution of the positive component for $\mathrm{A}(\sim 170 \mathrm{~ms})$ and AV ( $\sim 165 \mathrm{~ms})$ speech is shown in the bottom left and bottom middle, respectively. Difference topographies (AV-A) are shown for the negative component (top right) and positive components (bottom right). The same amplitude scale is used for all topographic maps and is indicated by the color bars.
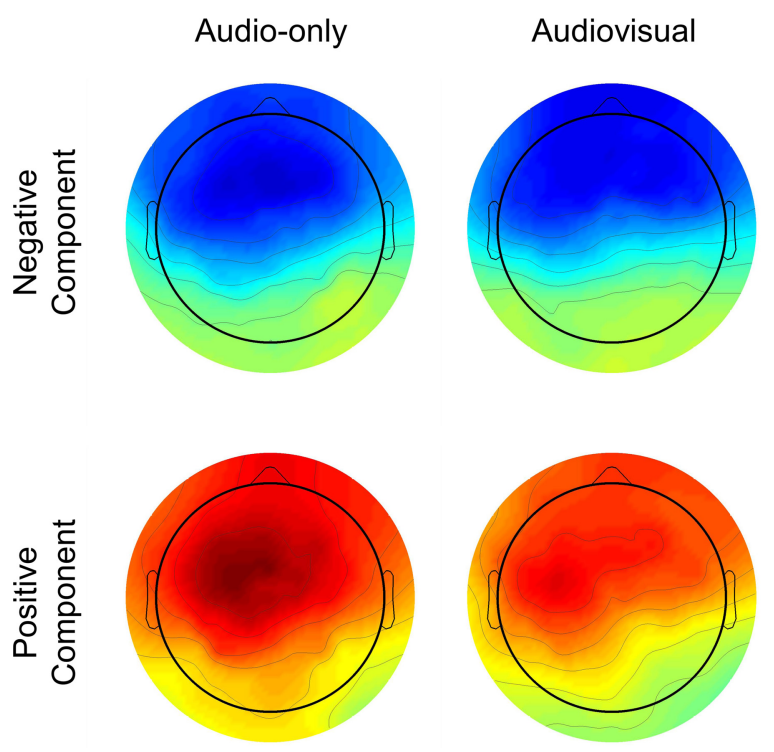

Difference
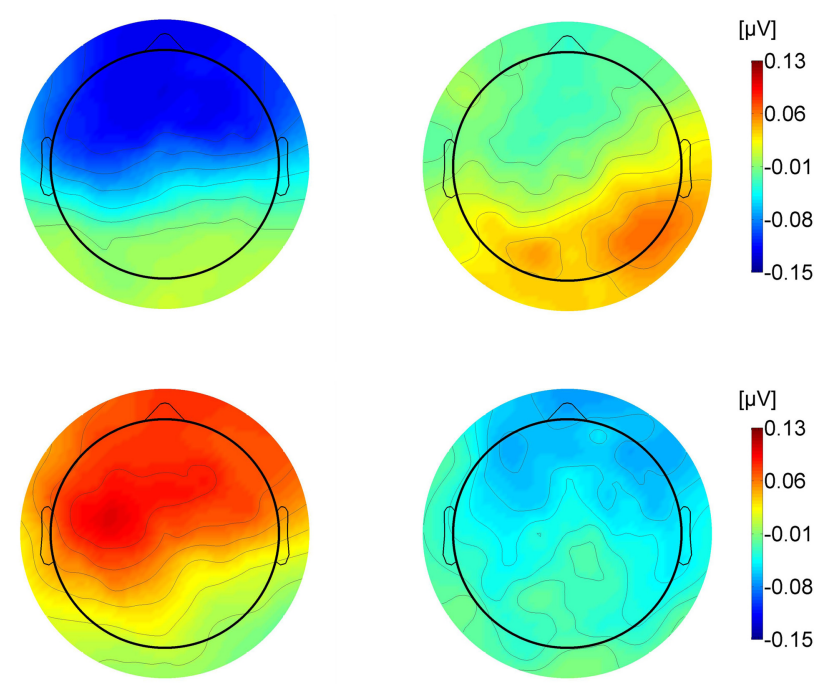

trated by the difference topographies (AV-A; Fig. 4), which display no signs of significant subadditive or supraadditive activity over the frontal electrodes shown in Fig. $2 A$.

To examine the possibility of common generators for the negative components of the A and AV TRFs even further, we performed source analysis using the BESA software package (Gräfelfing, Germany). Specifically, we fit two symmetrical dipoles to the negative components of the grand-averaged $\mathrm{A}$ and AV TRFs and compared their locations. We based our fits on 20-ms intervals around the component peaks in each condition (A interval: 100-120 ms; AV interval: 90-110 ms). The best fitting dipoles were located at very similar locations for the A and AV conditions (A Talairach coordinates: $x= \pm 40.5, y=$ $-25.8, z=15.7$; AV Talairach coordinates: $x= \pm 39.4, y=$ $-19.9, z=15)$. These fits explained 98.6 and $98.9 \%$ of the variance in the data for the A and AV TRFs, respectively. Indeed, fixing the dipoles to the best-fit locations for the A TRF and allowing their orientation to vary still explained $98.7 \%$ of the variance in the AV TRF, suggesting a large correspondence in the sources of both components.

Investigating nonlinear additive effects. As previously mentioned, many previous studies have interpreted nonlinear additive effects (i.e., differences between the amplitudes of AV and $\mathrm{A}+\mathrm{V}$ responses) as indices of multisensory integration (Arnal et al. 2009; Besle et al. 2004; Klucharev et al. 2003; Pilling 2009; Stekelenburg and Vroomen 2007; van Wassenhove et al. 2005). Given that some of these authors have suggested that latency shifts could contribute to these observed amplitude differences (Stekelenburg and Vroomen 2007) and that we have observed a clear latency shift when comparing the AV TRF with the A+V TRFs (Fig. 3), we checked for the existence of any amplitude differences between our response measures. Because, as mentioned above, the $\mathrm{V}$ TRF during the 0 - to 300-ms interval was not significantly different from baseline, we opted to check for possible amplitude effects by comparing the peak amplitude of both the negative and positive TRF components between the A and AV conditions. No difference was observed for either the negative component $(P=0.16$, paired $t$-test $)$ or the positive component $(P=0.12$, paired $t$-test).
This previous analysis was based solely on the amplitude of the peak TRF components, which actually occurred at different latencies. Another way of relating our AV data to the sum of the $\mathrm{A}$ and $\mathrm{V}$ data that does not rely on a specific choice of one response feature (i.e., the peak) nor on choosing a specific latency is stimulus reconstruction (Mesgarani et al. 2009). In the present case, this was done using temporally broad reconstruction filters $(0-250 \mathrm{~ms})$, which captured the dominant components of both the AV and $\mathrm{A}+\mathrm{V}$ conditions. As mentioned in the methods above, the different conditions could then be compared by measuring how accurately the speech envelope could be reconstructed from the corresponding multichannel neural response data. Reconstruction accuracy was based on the Pearson correlation between the reconstructed and original speech envelopes.

Figure 5 shows the distribution of single-trial reconstruction accuracy for the $\mathrm{A}, \mathrm{V}$, and $\mathrm{AV}$ conditions. In addition, for each subject and each video, we summed the corresponding A and $\mathrm{V}$ reconstructions and computed its correlation with the original speech envelope. The distribution of these correlation values is also shown. Considering first just the unimodal conditions, we found that the reconstruction accuracy of the $\mathrm{V}$ condition was significantly greater than $0\left(P=5 \times 10^{-28}\right.$, 1 -tailed $t$-test) with a mean $r$ value of 0.078 (SD 0.05). The fact that the speech envelope could be reconstructed from the $\mathrm{V}$ data suggests that there is a correlation between the dynamic changes in the visual and auditory stimuli (see next section for further discussion). As expected, the reconstruction accuracy for the A condition was significantly higher $\left(P=7.8 \times 10^{-19}\right.$, paired $t$-test) with a mean $r$ value of 0.145 (SD 0.056). To investigate potential nonlinear multisensory integrative processes in the AV condition, a statistical comparison was made between the $\mathrm{A}+\mathrm{V}$ and $\mathrm{AV}$ reconstructions. Mean reconstruction accuracies of 0.161 (SD 0.049) and 0.156 (SD 0.059) were calculated for the $\mathrm{AV}$ and $\mathrm{A}+\mathrm{V}$ conditions, respectively, and this difference was found not to be significant $(P=0.34$, paired $t$-test). That the speech envelope could not be reconstructed more accurately in the AV condition than in the A+V condition suggests a lack of any nonlinear additive multisensory effects in our paradigm. 

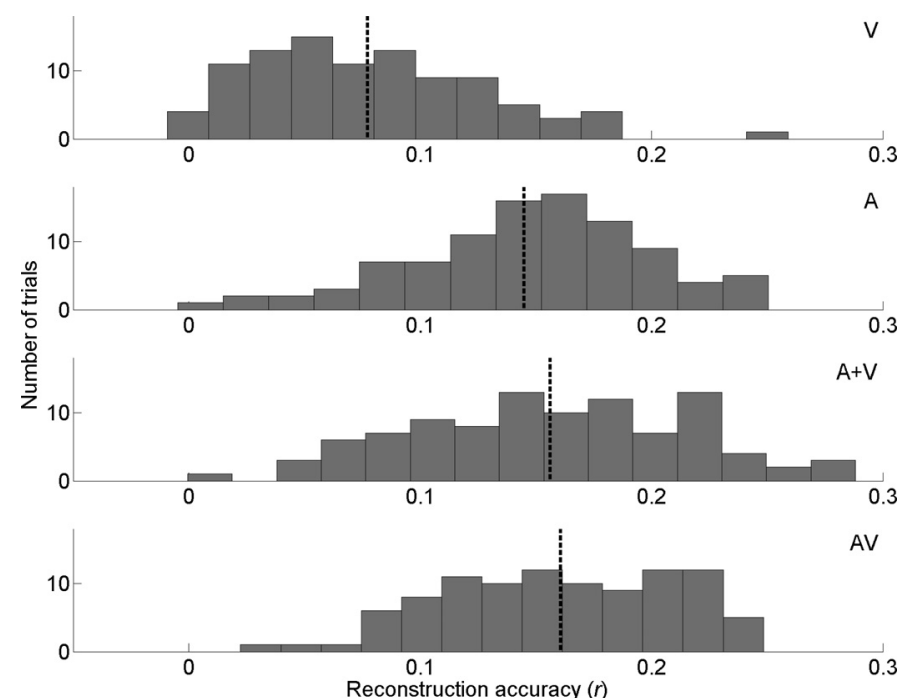

Fig. 5. Investigating nonlinear additivity to quantify multisensory interactions. Distributions of reconstruction accuracy (Pearson $r$ ) for the $\mathrm{V}, \mathrm{A}, \mathrm{A}+\mathrm{V}$, and $\mathrm{AV}$ conditions are shown. Each $\mathrm{A}+\mathrm{V}$ measure was obtained by summing the paired reconstructions from the $\mathrm{A}$ and $\mathrm{V}$ conditions and calculating the correlation between the resulting estimate and the original envelope. The dashed line corresponds to the mean value $(\mathrm{V}=0.078 ; \mathrm{A}=0.145 ; \mathrm{A}+\mathrm{V}=$ $0.156 ; \mathrm{AV}=0.161)$

Explaining the $V$ reconstructions. The fact that the speech envelope reconstructions from the $\mathrm{V}$ data were significantly greater than 0 suggests that certain characteristics of the video are correlated with the audio envelope. Based on the assumption that the contrast and luminance remained constant during each of our speech stimuli, our investigation focused on the motion within the video. Although previous studies have examined the relationship between the speech envelope and mouth movements (Chandrasekaran et al. 2009), it has also been shown that even head movements are highly correlated with auditory speech (Munhall et al. 2004; Munhall and Vatikiotis-Bateson 1998). We, therefore, examined the frameto-frame motion flow and its relationship with the auditory envelope for each of the seven videos used in this experiment.
For each frame, a matrix of motion vectors was calculated using an Exhaustive Search (ES) block matching algorithm (Barjatya 2004). A measure of motion flow was obtained by calculating the sum of all motion vector lengths of each frame (Bartels et al. 2008). The envelope of the auditory signal was downsampled from $44.1 \mathrm{kHz}$ to $27.97 \mathrm{~Hz}$ to match the frame rate of the video.

Figure $6 A$ plots the broadband speech envelope and the frame-to-frame motion flow for an 8-s segment of one of the seven videos used. This plot shows that the motion signal precedes the audio signal at the start of every sentence. A cross-correlation of these signals (Fig. 6B) across the entire duration of the video revealed a maximal correlation at a nonzero lag. This effect was found in all seven videos with the motion preceding the audio on average by $\sim 140 \mathrm{~ms}$, consistent with previously reported visual-audio lags in speech stimuli (Chandrasekaran et al. 2009; Pilling 2009; Stekelenburg and Vroomen 2007). The audio and motion signals were significantly correlated $(P<0.05)$ for six of the seven videos. If we assume that the processing of our visual motion signal is represented in the EEG (which is not necessarily true), then this correlation between auditory and visual speech (at least partially) explains how an estimate of the auditory envelope could be reconstructed from the $\mathrm{V}$ data with an accuracy that was significantly greater than chance. Other features of the visual stimulus that also correlated with the audio envelope and that are represented in the EEG signal may also have contributed.

Although we did not see any significant TRF measure in response to the $\mathrm{V}$ condition (Fig. $2 A$ ), the broadly distributed lag between the visual and audio streams, peaking at $\sim 140 \mathrm{~ms}$, suggests that a V TRF may occur at some earlier time lag that was not visible in our original TRF analysis. To investigate this, we conducted an additional TRF analysis over a broader time window ( -300 to $300 \mathrm{~ms}$ ) at frontocentral electrode $\mathrm{Fz}$ (Fig. 6C) and occipitocentral electrode $\mathrm{Oz}$ (Fig. 6D). The TRFs calculated in this way did not display any large components. This is due to the fact that the TRF analysis is based on the
A 1
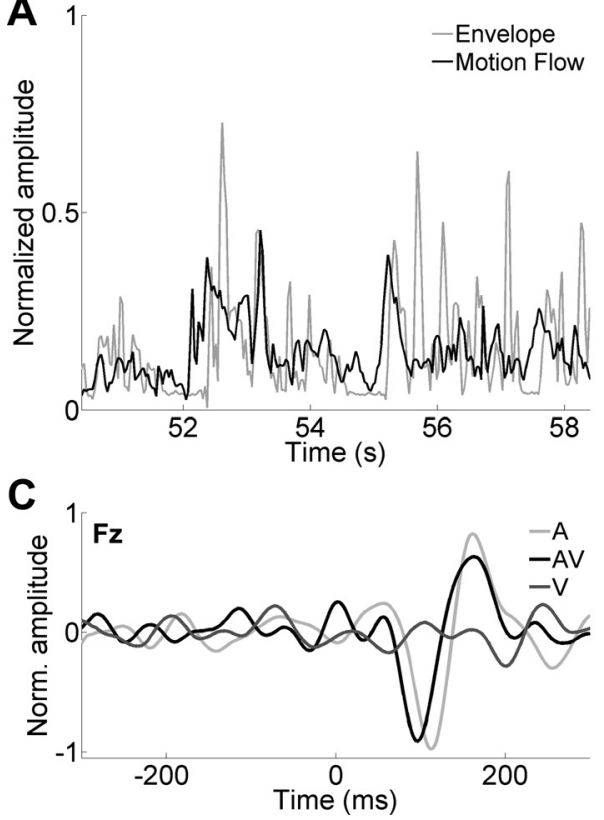

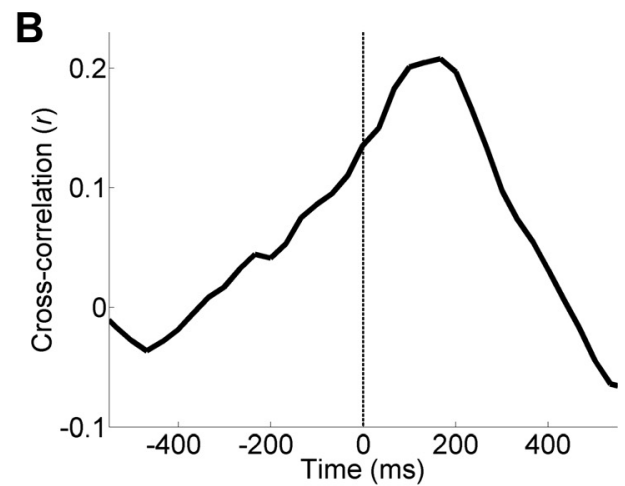

D

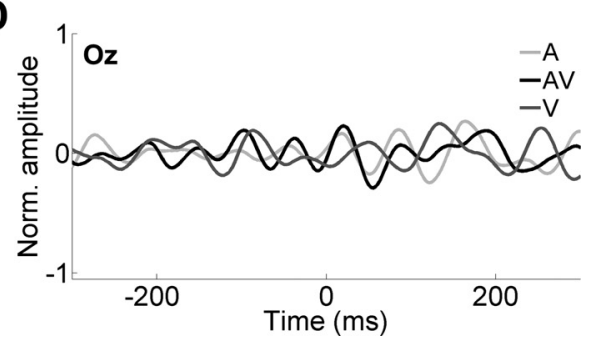

Fig. 6. Temporal lag between visual and auditory speech. A: auditory speech envelope (gray trace) and frame-to-frame motion flow (black trace) for an 8-s time segment of 1 of the videos used in the experiment. There is a clear correspondence between the auditory envelope and the motion flow. The temporal lag is also very evident at the start of each sentence. $B$ : cross-correlation of the same envelope and motion signals. The dotted line represents a 0 time lag relative to the motion signal. $C$ : grand-average TRFs calculated at frontocentral site $\mathrm{Fz}$ for an extended time window ( -300 to $300 \mathrm{~ms}$ ). There is no evidence of visual-evoked TRFs at these earlier lags. Norm., normalized. $D$ : grand-average TRFs calculated at occipitocentral site $\mathrm{Oz}$ over the same time window. Again there is no evidence, even at this occipital site, of any visual-evoked TRFs. 
audio envelope and the fact that the audio envelope and motion signals are correlated with each other over a broad range of nonzero lags (Fig. 6B). This weak representation of the visual motion in the EEG signal highlights that the approach we have used is effective at acquiring response measures (i.e., the TRFs) dominated by auditory cortex in relative isolation.

\section{DISCUSSION}

We have shown a marked shortening of auditory response latency to natural continuous speech when it is accompanied by congruent visual speech. In addition, we have found no evidence of any nonlinear additive multisensory effects during natural AV speech. We contend that this provides important insight into the issue of latency shifts vs. amplitude effects that has been noted in previous work examining ERPs derived to discrete stimuli. In particular, the demonstrated latency shift is likely a correlate of an underlying mechanism that is fundamental to enhanced comprehension during AV speech. Specifically, the fact that our latency effect is seen on the early $(\sim 100$ ms) negative TRF component, a latency that has been linked with analysis of acoustic-phonetic speech features (Salmelin 2007), suggests that it may index an earlier synthesis of low-level audio features into a coherent stream. This would likely allow a longer time frame for further processing before extraction of semantic information from that stream, which has been suggested to occur after $200 \mathrm{~ms}$ (Salmelin 2007).

Disentangling multisensory latency and amplitude effects. Our findings fit well with a number of previous EEG/MEG studies that have proposed that visual input can speed up the processing of discrete auditory syllables (Arnal et al. 2009; Stekelenburg and Vroomen 2007; van Wassenhove et al. 2005). Specifically, these studies analyzed time-locked average ERPs/event-related fields (ERFs) and showed that the latency of particular component peaks was shortened for AV speech relative to A speech. It was even reported that the magnitude of this latency shortening was proportional to the degree to which the visual signal predicted the subsequent auditory stimulus (Arnal et al. 2009; van Wassenhove et al. 2005). An interesting feature of the ERPs/ERFs reported in these studies was that the $\mathrm{AV}$ and A responses onset at the same latency and increased with the same slope but that the AV responses had lower amplitude. Thus it was suggested that the peak latency shortening may simply be an artifact of this lower amplitude (Stekelenburg and Vroomen 2007). Although, as mentioned above, it is difficult to disentangle putative latency effects from the putative nonlinear summation of time-locked visual and auditory responses to discrete multimodal stimuli, our data appear to validate the existence of a latency effect in these studies. This is particularly true given that our study has utilized a data analysis approach that has produced A and AV responses that are likely dominated by auditory cortex with minimal direct contributions to the AV TRF from visual cortical activity. This is because the TRF method is based specifically on an assumption of a linear relationship between the audio envelope and the EEG. Although the global motion in the video is correlated with the audio envelope, it is correlated in a temporally broad, imprecise, and variable way (Fig. $6 B$ ). As such, regressing the EEG data against the envelope of the speech did not produce temporally precise TRF components in the V condition, although it still allowed reconstruc- tion of the speech envelope from the $\mathrm{V}$ condition data. This implies that the sharp TRF components in the AV condition are likely to be largely generated by activity in auditory cortex. The similarities in topographic distribution and source locations between the A and AV conditions support this claim. This suggests that the differing latencies of the negative components in the A and AV conditions reflects a genuine speed-up of processing in auditory cortex, a finding that is well-supported by recent research in monkey auditory cortex (Chandrasekaran et al. 2013).

Previous EEG-based studies have compared the amplitude of ERPs evoked by AV stimuli to an additive model $(\mathrm{A}+\mathrm{V})$ based on the assumption that any differences may be attributed to multisensory interactions (Besle et al. 2004; Klucharev et al. 2003). What are the implications of the observed latency shift for such an approach? Each ERP component, as it is measured on the scalp, is the result of the summed activation of several neural generators, each of which may become active at different latencies (Luck 2004). For example, two separate ERPs that differ in both amplitude and latency may only appear to differ in amplitude because a certain contribution to one of the ERPs has a greater temporal overlap with activity from other sources. Thus the interpretation of a nonlinear additive effect appears to be very difficult to validate if possible underlying latency shifts have been ignored. This is highlighted by the lack of any nonlinear additive effects in our analysis of either peak amplitude or stimulus reconstruction accuracy. It should be noted that, because we assume a linear relationship between the speech envelope and the EEG, it is likely that our TRF measures are not sensitive to nonlinear lateral and feedback contributions to speech processing. It could be that some of these contributions may show amplitude effects that are seen in the ERP. That said, the lack of any amplitude effects on the TRFs in this study is in line with recent work on AV vocalizations in monkey auditory cortex that showed no firing rate amplitude effects with the addition of a visual input (Chandrasekaran et al. 2013). Also, the lack of any multisensory amplitude effects may be due to the fact that our paradigm involved an easy-to-hear listening environment in which multimodal speech would be no easier to understand than unimodal speech (Ross et al. 2007a). Future work is planned to investigate whether AV stimulus reconstructions perform significantly better than those based on the sum of A and V reconstructions for a speech-in-noise paradigm, although, interestingly, the recent monkey work also showed no multisensory amplitude effects when the signal-to-noise ratio of the audio vocalizations was reduced (Chandrasekaran et al. 2013).

Possible mechanisms of temporal facilitation. The most likely cause of the latency shift that we observe in our AV data is the precedence of visual speech, a well-known phenomenon. Stekelenburg and Vroomen (2007) showed that this effect is also present during ecologically valid nonspeech stimuli if the visual stimulus preceded the auditory stimulus (e.g., handclapping) but not if it did not (e.g., tearing paper). There are several possible mechanisms through which the preceding visual input could cause this effect on auditory processing. One plausible process relates to the correlation between the speech envelope and visual motion. This correlation suggests that anticipatory visual motion could produce phasic variations in visual cortical activity that are relayed to auditory cortex and that correlate with the amplitude envelope of the subsequent auditory speech. 
This notion fits with previous work showing that the phase of oscillations in auditory cortex tracks the temporal structure of continuous visual speech (Luo et al. 2010) and fMRI data that has shown the source of the visual facilitation of speech arises from motion-sensitive cortex (Arnal et al. 2009).

Based on our EEG data, it is not possible to determine definitively the specific mechanisms or pathways by which visual activity might impact on auditory processing. It has previously been suggested this interaction might be driven by relatively discrete visual landmarks (e.g., the onset of facial articulatory movements) that may elicit a phase reset of ongoing low-frequency oscillations in auditory cortex such that the arrival of the corresponding auditory syllable coincides with a high excitability phase of the auditory neuronal population (Kayser et al. 2008; Schroeder et al. 2008). While compelling evidence has been shown for multisensory phase reset in humans using discrete stimuli (Mercier et al. 2013), the efficacy of such a mechanism in the context of continuous speech seems like it would necessitate prior knowledge about which part of an upcoming syllable was most important. For example, consider the syllable pairs bat vs. vat, bat vs. bet, and bat vs. bad. Discriminating between syllables within each of these pairs is conducted using a different part of the syllable (start, middle, and end, respectively). How a phase reset may align the high excitability phase of the auditory neuronal population with the most important part of the syllable before that syllable arrives is unclear. This is particularly true where phase resets are occurring at a nonperiodic rate in line with the frequency of the arrival of natural speech syllables (e.g., 4-7 Hz).

Another possible multisensory mechanism, which has previously been proposed in the context of the synthesis of auditory objects, is that the particular neural populations may be sensitive to temporal correlations between the speech envelope and visual motion, leading to them becoming integrated into a coherent multisensory speech object. Recent work has shown that cortical entrainment to the speech envelope is not just representative of bottom-up envelope-tracking but that it may index an analysis-by-synthesis approach to speech processing (Ding et al. 2013). This framework proposes that speech is first analyzed by breaking it up into constituent spectrotemporal channels and that auditory objects are synthesized from those channels that modulate together in a temporally coherent manner. Within such a framework, Ding et al. (2013) have shown that cortical entrainment to speech depends on the detailed spectrotemporal fine structure of speech and that, as such, the cortical entrainment to the speech envelope may represent the temporally coherent fluctuations of the many spectrotemporal features that make up the speech signal. This fits with a theory postulating that, in auditory scene analysis, multifeature auditory sources are segregated into perceptual streams based on the temporal coherence of such acoustic features (Shamma et al. 2011). In keeping with previous work espousing a correlated mode of processing for AV speech (Campbell 2008), we postulate that motion in the visual stream, being correlated with the dynamics of the auditory features, results in the visual signal being bound to the audio features to form a multisensory object. In terms of intelligibility, this partially explains the benefit conferred by the presence of visual speech in adverse acoustic conditions. The fact that the visual motion precedes the auditory features may mean that the binding of auditory features occurs earlier and that this syn- thesis is reflected in a shorter latency of our envelope-tracking measure. Such a suggestion also seems to be compatible with the idea of predictive coding (Arnal et al. 2009; van Wassenhove et al. 2005). Of course, the TRF we observe is still derived from the envelope of the heard speech. Thus the latency shift we obtain $(\sim 10 \mathrm{~ms})$ is but a small fraction of the actual amount by which the video preceded the audio $(>100$ $\mathrm{ms}$ ). This discrepancy in timings is also likely to be a result of the time it takes for motion information from the visual cortex to have an impact on auditory processing.

In terms of what specific neural populations might facilitate the binding of temporally coherent visual and audio speech, one candidate region is the superior temporal sulcus, which has previously been linked with multisensory speech processes (Beauchamp et al. 2004). Indeed, recent research has shown evidence for neural computations in this area that underpin auditory figure-ground segregation using stimuli that display periods of temporal coherence across multiple frequency channels (Teki et al. 2011). Of course, it remains a possibility that AV speech interactions could occur through direct connections (Schroeder et al. 2008). The latency of our TRF effects $(\sim 100$ ms) suggests that this may indeed be true (Arnal et al. 2009). Further research using alternative brain recording methods may elucidate these issues.

Comparisons with other findings and future applications. Our findings are somewhat in contrast with recent MEG work that also used continuous auditory speech to examine the effects of visual input on TRFs acquired to A and AV speech (Zion-Golumbic et al. 2013). This study reported no latency effects on TRFs that were dominated by a single peak at $\sim 50$ ms, which is considerably earlier than the components that we have focused on here. This difference may simply be a result of the sensitivities of EEG and MEG to different processes within the auditory system. A possible explanation for the lack of a latency effect on such an early component is that it is so early that it may only index the analysis phase of the analysis-bysynthesis process, i.e., the phase wherein the basic acoustic features of the auditory signal are extracted subcortically (Nelken 2008). Processing at this early stage may be unaffected by the dynamics of a simultaneously presented visual input.

Although it will certainly require methods complementary to human EEG to determine the details of the neural mechanisms underlying AV speech integration, we suggest that the latency effect that we have identified may index these underlying mechanisms. This robust index, acquired using natural stimuli and a relatively inexpensive and accessible technology, may prove useful in research with clinical populations in which altered multisensory (AV) processing has been reported, e.g., dyslexia (Hairston et al. 2005), autism (Brandwein et al. 2013), and schizophrenia (Ross et al. 2007b).

\section{ACKNOWLEDGMENTS}

We thank James O'Sullivan, Gerard Loughnane, and Fiona Newell for critical discussion and reading of the manuscript.

\section{GRANTS}

This work was supported by the Irish Higher Education Authority Graduate Research Education Programme in Engineering and by a grant from Science Foundation Ireland (09-RFP-NES2382). 


\section{DISCLOSURES}

No conflicts of interest, financial or otherwise, are declared by the author(s).

\section{AUTHOR CONTRIBUTIONS}

M.J.C. and E.C.L. conception and design of research; M.J.C. performed experiments; M.J.C. analyzed data; M.J.C. and E.C.L. interpreted results of experiments; M.J.C. prepared figures; M.J.C. drafted manuscript; M.J.C. and E.C.L. edited and revised manuscript; M.J.C. and E.C.L. approved final version of manuscript.

\section{REFERENCES}

Arnal LH, Morillon B, Kell CA, Giraud AL. Dual neural routing of visual facilitation in speech processing. J Neurosci 29: 13445-13453, 2009.

Barjatya A. Block matching algorithms for motion estimation. IEEE Trans Evol Comput 8: 225-239, 2004.

Bartels A, Zeki S, Logothetis NK. Natural vision reveals regional specialization to local motion and to contrast-invariant, global flow in the human brain. Cereb Cortex 18: 705-717, 2008.

Beauchamp MS, Lee KE, Argall BD, Martin A. Integration of auditory and visual information about objects in superior temporal sulcus. Neuron 41: 809-824, 2004.

Besle J, Fort A, Delpuech C, Giard MH. Bimodal speech: early suppressive visual effects in human auditory cortex. Eur J Neurosci 20: 2225-2234, 2004.

Brandwein AB, Foxe JJ, Butler JS, Russo NN, Altschuler TS, Gomes H, Molholm S. The development of multisensory integration in high-functioning autism: high-density electrical mapping and psychophysical measures reveal impairments in the processing of audiovisual inputs. Cereb Cortex 23 . 1329-1341, 2013.

Campbell R. The processing of audio-visual speech: empirical and neural bases. Philos Trans R Soc Lond B Biol Sci 363: 1001-1010, 2008.

Chandrasekaran C, Lemus L, Ghazanfar AA. Dynamic faces speed up the onset of auditory cortical spiking responses during vocal detection. Proc Natl Acad Sci USA 110: E4668-E4677, 2013.

Chandrasekaran C, Trubanova A, Stillittano S, Caplier A, Ghazanfar AA. The natural statistics of audiovisual speech. PLoS Comput Biol 5: e1000436, 2009.

Delorme A, Makeig S. EEGLAB: an open source toolbox for analysis of single-trial EEG dynamics including independent component analysis. $J$ Neurosci Methods 134: 9-21, 2004.

Di Russo F, Pitzalis S, Spitoni G, Aprile T, Patria F, Spinelli D, Hillyard SA. Identification of the neural sources of the pattern-reversal VEP. Neuroimage 24: 874-886, 2005.

Ding N, Chatterjee M, Simon JZ. Robust cortical entrainment to the speech envelope relies on the spectro-temporal fine structure. Neuroimage. First published November 2, 2013; doi:10.1016/j.neuroimage.2013.10.054.

Hairston WD, Burdette JH, Flowers DL, Wood FB, Wallace MT. Altered temporal profile of visual-auditory multisensory interactions in dyslexia. Exp Brain Res 166: 474-480, 2005.

Kayser C, Petkov CI, Logothetis NK. Visual modulation of neurons in auditory cortex. Cereb Cortex 18: 1560-1574, 2008.

Klucharev V, Mottonen R, Sams M. Electrophysiological indicators of phonetic and non-phonetic multisensory interactions during audiovisual speech perception. Brain Res Cogn Brain Res 18: 65-75, 2003.

Lalor EC, Foxe JJ. Neural responses to uninterrupted natural speech can be extracted with precise temporal resolution. Eur J Neurosci 31: 189-193, 2010

Lalor EC, Power AJ, Reilly RB, Foxe JJ. Resolving precise temporal processing properties of the auditory system using continuous stimuli. $J$ Neurophysiol 102: 349-359, 2009.

Luck S. Ten simple rules for designing and interpreting ERP experiments. In: Event-Related Potentials: A Methods Handbook, edited by Handy TC. Cambridge, MA: MIT Press, 2004.
Luo H, Liu ZX, Poeppel D. Auditory cortex tracks both auditory and visual stimulus dynamics using low-frequency neuronal phase modulation. PLoS Biol 8: e1000445, 2010.

Mercier MR, Foxe JJ, Fiebelkorn IC, Butler JS, Schwartz TH, Molholm S. Auditory-driven phase reset in visual cortex: human electrocorticography reveals mechanisms of early multisensory integration. Neuroimage 79: 19-29, 2013.

Mesgarani N, David SV, Fritz JB, Shamma SA. Influence of context and behavior on stimulus reconstruction from neural activity in primary auditory cortex. J Neurophysiol 102: 3329-3339, 2009.

Millman RE, Prendergast G, Hymers M, Green GG. Representations of the temporal envelope of sounds in human auditory cortex: can the results from invasive intracortical "depth" electrode recordings be replicated using noninvasive MEG “virtual electrodes"? Neuroimage 64: 185-196, 2013.

Molholm S, Ritter W, Murray MM, Javitt DC, Schroeder CE, Foxe JJ. Multisensory auditory-visual interactions during early sensory processing in humans: a high-density electrical mapping study. Brain Res Cogn Brain Res 14: 115-128, 2002.

Munhall KG, Jones JA, Callan DE, Kuratate T, Vatikiotis-Bateson E. Visual prosody and speech intelligibility: head movement improves auditory speech perception. Psychol Sci 15: 133-137, 2004.

Munhall KG, Vatikiotis-Bateson E. The moving face during speech communication. In: Hearing by Eye, Part 2: The Psychology of Speechreading and Audiovisual Speech, edited by Campbell R, Dodd B, and Burnham D. London: Taylor \& Francis, Psychology Press, 1998, p. 123.

Murray MM, Brunet D, Michel CM. Topographic ERP analyses: a step-bystep tutorial review. Brain Topogr 20: 249-264, 2008.

Nelken I. Processing of complex sounds in the auditory system. Curr Opin Neurobiol 18: 413-417, 2008.

Nourski KV, Reale RA, Oya H, Kawasaki H, Kovach CK, Chen HM, Howard MA, Brugge JF. Temporal envelope of time-compressed speech represented in the human auditory cortex. J Neurosci 29: 15564-15574, 2009

Pilling M. Auditory event-related potentials (ERPs) in audiovisual speech perception. J Speech Lang Hear Res 52: 1073-1081, 2009.

Reisberg D, McLean J, Goldfield A. Easy to hear but hard to understand: a lip-reading advantage with intact auditory stimuli. In: Hearing by Eye: The Psychology of Lip-Reading, edited by Dodd B and Campbell R. Hillsdale, NJ: Lawrence Erlbaum Associates, 1987, p. 97-114.

Ross LA, Saint-Amour D, Leavitt VM, Javitt DC, Foxe JJ. Do you see what I am saying? Exploring visual enhancement of speech comprehension in noisy environment. Cereb Cortex 17: 1147-1153, 2007a.

Ross LA, Saint-Amour D, Leavitt VM, Molholm S, Javitt DC, Foxe JJ. Impaired multisensory processing in schizophrenia: deficits in the visual enhancement of speech comprehension under noisy environmental conditions. Schizophr Res 97: 173-183, 2007b.

Salmelin R. Clinical neurophysiology of language: the MEG approach. Clin Neurophysiol 118: 237-254, 2007.

Schroeder CE, Lakatos P, Kajikawa Y, Partan S, Puce A. Neuronal oscillations and visual amplification of speech. Trends Cogn Sci 12: 106113, 2008.

Shamma SA, Elhilali M, Micheyl C. Temporal coherence and attention in auditory scene analysis. Trends Neurosci 34: 114-123, 2011.

Stekelenburg JJ, Vroomen J. Neural correlates of multisensory integration of ecologically valid audiovisual events. J Cogn Neurosci 19: 1964-1973, 2007.

Sumby WH, Pollack I. Visual contribution to speech intelligibility in noise. J Acoust Soc Am 26: 212-215, 1954.

Teki S, Chait M, Kumar S, von Kriegstein K, Griffiths TD. Brain bases for auditory stimulus-driven figure-ground segregation. J Neurosci 31: 164$171,2011$.

van Wassenhove V, Grant KW, Poeppel D. Visual speech speeds up the neural processing of auditory speech. Proc Natl Acad Sci USA 102: 11811186, 2005.

Zion-Golumbic EM, Cogan GB, Schroeder CE, Poeppel D. Visual input enhances selective speech envelope tracking in auditory cortex at a "cocktail party". J Neurosci 33: 1417-1426, 2013. 